ARTICLE OPEN

\title{
Visualization of subnanometric phonon modes in a plasmonic nano-cavity via ambient tip-enhanced Raman spectroscopy
}

Maria Vanessa Balois (D) ${ }^{1 *}$, Norihiko Hayazawa ${ }^{1,2 *}$, Satoshi Yasuda $\mathbb{D}^{3}$, Katsuyoshi Ikeda (D) ${ }^{4}$ Bo Yang ${ }^{2,7}$, Emiko Kazuma ${ }^{2}$, Yasuyuki Yokota $\mathbb{i D}^{2}$, Yousoo $\mathrm{Kim}^{2}$ and Takuo Tanaka, ${ }^{1,5,6 *}$

Phonons provide information on the physicochemical properties of a crystalline lattice from the material's vibrational spectrum. Optical phonons, in particular, can be probed at both micrometre and nanometre scales using light-based techniques, such as, micro-Raman and tip-enhanced Raman spectroscopy (TERS), respectively. Selection rules, however, govern the accessibility of the phonons and, hence, the information that can be extracted about the sample. Herein, we simultaneously observe both allowed and forbidden optical phonon modes of defect-free areas in monolayer graphene to study nanometre scale strain variations and plasmonic activation of the Raman peaks, respectively, using our home-built TERS system in ambient. Through TERS imaging, strain variations and nanometre-sized domains down to $5 \mathrm{~nm}$ were visualised with a spatial resolution of $0.7 \mathrm{~nm}$. Moreover, such subnanometric confinement was found to activate not only the $D$ and $D^{\prime}$ forbidden phonon modes but also their $D+D^{\prime}$ combination mode. With our TERS in ambient system, the full phonon characterisation of defect-free graphene and other 2D nanomaterials is now possible, which will be useful for subnanometre strain analysis and exploring the inherent properties of defect-free materials.

npj 2D Materials and Applications (2019)3:38

; https://doi.org/10.1038/s41699-019-0121-7

\section{INTRODUCTION}

Tip-enhanced Raman spectroscopy (TERS) can simultaneously provide the high spatial resolution and chemical sensitivity needed for materials and device characterisation at the nanoscale through the highly confined enhanced electromagnetic field generated within a plasmonic nano-cavity. ${ }^{1-14}$ It has recently been demonstrated that TERS systems based on a scanning tunnelling microscope (STM) can obtain impressive images and information with subnanometre resolution, ${ }^{10-12}$ but these systems are restricted to special extreme environments (e.g. ultra-high vacuum and low temperature) that are not suitable to study the asfabricated electrical and mechanical properties of devices. This is of particular importance for graphene-based devices, since graphene's electronic properties can be engineered via strain ${ }^{15}$ typically induced at the graphene-substrate interface that have different thermal expansion coefficients. To broaden the applications of STM-TERS for characterising room-temperature operating devices and environment sensitive samples, high spatial resolution in ambient is vital. This need was addressed by our group in 2014, where we obtained a resolution of $1.7 \mathrm{~nm}$ while conducting chemical analysis of carbon nanotubes using our STM-TERS system in ambient. ${ }^{13}$ Herein, we go even further down to a subnanometre resolution of $0.7 \mathrm{~nm}$-the highest reported so far for TERS in ambient conditions, to our knowledge-to directly observe and study nanometre-scale strain variations in defect-free graphene through the detection of allowed and forbidden phonon modes.

In this work, we demonstrate subnanometre spatial resolution via TERS imaging of defect-free areas of monolayer graphene and the observation of the forbidden phonon modes, $D, D^{\prime}$, and, the unprecedented observation of the combination mode, $D+D^{\prime}$, using TERS. These modes are forbidden in pristine graphene due to the absence of defects and, instead, are activated by a plasmonmediated process ${ }^{16}$ or "field gradient effect" (FGE), a phenomenon that can alter both Raman ${ }^{17}$ and electronic excitation ${ }^{18}$ selection rules of a probed sample. FGE is due to the strong field gradient caused by the evanescent nature of the highly confined electromagnetic field at the tip apex. Using subnanometre resolution TERS imaging and TERS data, we visualise and analyse the local strain variations in defect-free areas of graphene that are reported to affect the maximum observed carrier mobility in highquality graphene. ${ }^{19}$ So far, these local strain fluctuations have not been directly observed due to the diffraction limit and are mainly inferred through the spectral width broadening of the $2 D$ peak measured via conventional Raman spectroscopy (microRaman). ${ }^{19,20}$ Lastly, we show the experimental TERS observation of the theoretically predicted ${ }^{21}$ splitting of the $D^{\prime}$ peak and discuss the anisotropy in subnanometric strain.

\section{RESULTS}

Monolayer graphene on $\mathrm{Au}(111)$

Our sample is a monolayer of graphene that was grown directly on an $\mathrm{Au}(111)$ single crystal substrate $(\mathrm{Gr} / \mathrm{Au}(111))$ via atmospheric chemical vapour deposition (AP-CVD). ${ }^{22}$ We used microRaman spectroscopy and STM topography measurements to examine the condition of the monolayer graphene on the $\mathrm{Au}(111)$ substrate. From micro-Raman measurements $(\lambda=532 \mathrm{~nm}$, see

\footnotetext{
${ }^{1}$ Innovative Photon Manipulation Research Team, RIKEN Center for Advanced Photonics, 2-1 Hirosawa, Wako, Saitama 351-0198, Japan. ${ }^{2}$ Surface and Interface Science Laboratory, RIKEN Cluster for Pioneering Research, 2-1 Hirosawa, Wako, Saitama 351-0198, Japan. ${ }^{3} J a p a n$ Atomic Energy Agency, Sector of Nuclear Science Research, Advanced Science Research Center, Tokai, Naka, Ibaraki 319-1195, Japan. ${ }^{4}$ Department of Physical Science and Engineering, Nagoya Institute of Technology, Gokiso, Showa, Nagoya 466-8555,

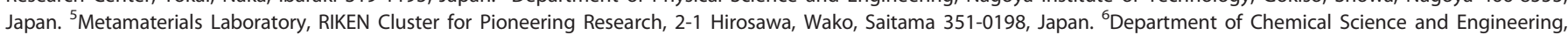
Tokyo Institute of Technology, Yokohama, Kanagawa 226-8503, Japan; ${ }^{7}$ Present address: School of Science, Xijing University, No. 1 , Xijing Road, 710123 Xi'an, China. *email: mariavanessa.balois@riken.jp; hayazawa@riken.jp; t-tanaka@riken.jp
} 

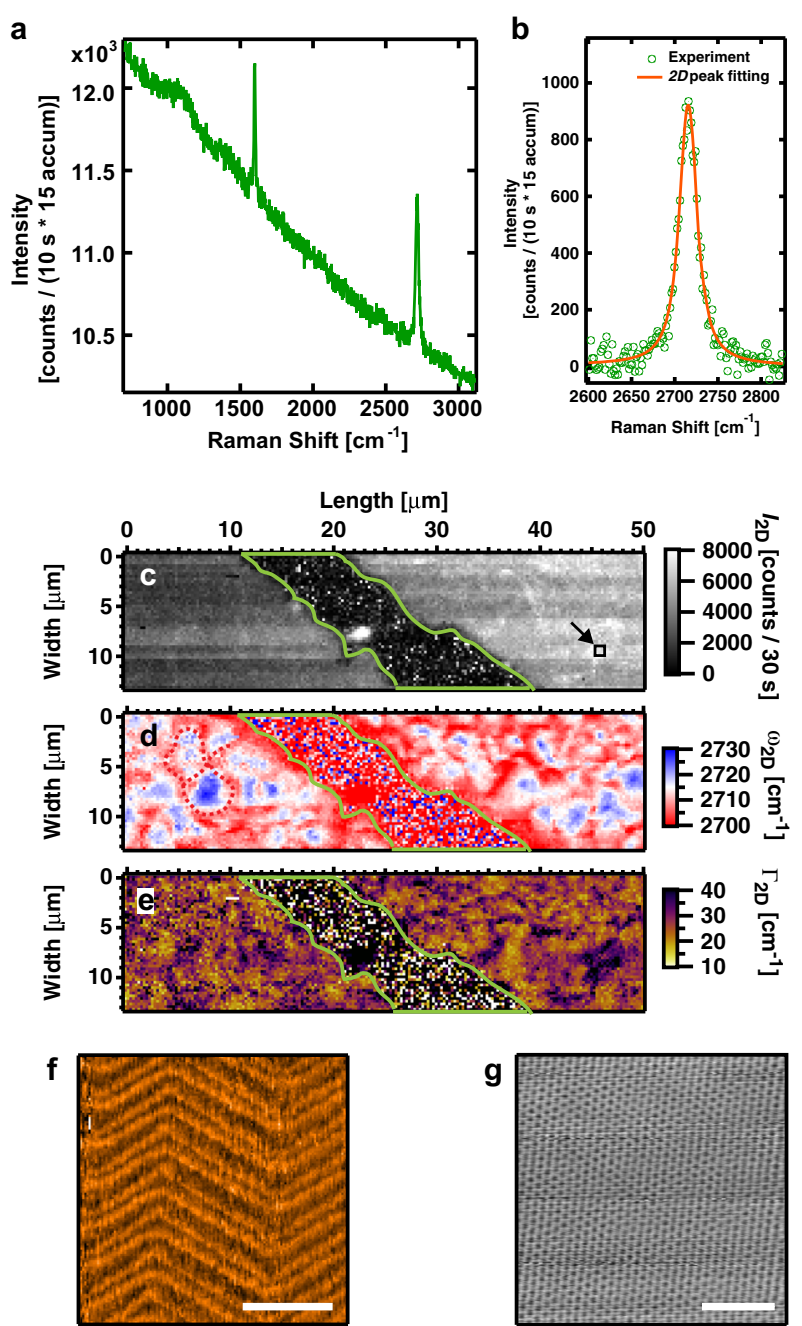

Fig. 1 Characterisation of $\mathrm{Gr} / \mathrm{Au}(111)$ via micro-Raman spectroscopy and STM. a Representative micro-Raman spectrum from the experiment area showing only $G$ and $2 D$ bands. Absence of $D$ band $\left(\sim 1340 \mathrm{~cm}^{-1}\right)$ indicates the probed area is defect free. b Experimental and single Voigt fitted data of $2 D$ band from $\mathbf{a}$, with a spectral width of $21.0 \mathrm{~cm}^{-1}$, indicating monolayer graphene is present. $^{23}$ Background from the spectrum was subtracted prior to fitting. Micro-Raman mapping of $2 D$ band's c integrated intensity $\left(I_{2 D}\right)$, d Raman shift $\left(\omega_{2 D}\right)$ and e spectral width $\left(\Gamma_{2 D}\right)$. Single Voigt fitting was used with constant Gaussian component of $8.5 \mathrm{~cm}^{-1}$ and only the Lorentzian component was extracted. Cracks in the monolayer graphene due to thermal expansion are enclosed by green lines. Wrinkles (red dashed lines) with red-shifted Raman peaks divide the graphene into micro-domains whose Raman peak positions are blue-shifted. Excitation laser with $\lambda=532 \mathrm{~nm}$ (NA= $0.9, \times 100)$ was used for micro-Raman experiments. Black box in c indicated by the arrow is the scan area used for STM-TERS experiments. f STM image of $\mathrm{Au}(111)$ herringbone structure preserved underneath the monolayer graphene on the $\mathrm{Gr} / \mathrm{Au}(111)$ (modified Au tip; tunnelling current: $0.5 \mathrm{nA}$; bias voltage: $300 \mathrm{mV}$ ). Scale bar indicates $10 \mathrm{~nm}$. g Atomic resolution STM current image of $\mathrm{Gr} / \mathrm{Au}(111)$ defect-free honeycomb structures (Pt/Ir tip; tunnelling current: $2 \mathrm{nA}$; bias voltage: $5 \mathrm{mV}$ ). Scale bar indicates $2 \mathrm{~nm}$

Methods), we verified that majority of the graphene was (1) defect-free upon observing only the $G$ band $\left(\omega_{\mathrm{G}, \mathrm{Au}, 532}=\right.$ $\left.1599 \mathrm{~cm}^{-1}\right)$ and the $2 D$ band $\left(\omega_{2 D, A u, 532}=2715.6 \mathrm{~cm}^{-1}\right)$, as shown in Fig. $1 \mathrm{a}$, and (2) monolayer based on the narrow spectral width of the $2 D$ band $\left(\Gamma_{2 D, A u, 532}=21.0 \mathrm{~cm}^{-1}\right.$, Fig. $\left.1 \mathrm{~b}\right) .{ }^{23}$ We rarely observed a weak $D$-band in several areas mostly located near the edge of the substrate (see Supplementary Fig. 1), and we can easily select the areas without any $D$-band near the centre of the substrate for the measurements presented in this paper. Aside from the Raman peaks, a broad background was also observed in all measurements and may originate from electronic Raman scattering in $\mathrm{Gr} / \mathrm{Au}(111){ }^{24}$ The observed peak positions of the $G$ and the $2 D$ Raman bands are considerably blue-shifted compared to monolayer graphene on $\mathrm{SiO}_{2} \quad\left(\omega_{\mathrm{G}, \mathrm{SiO} 2,532}=1580.8 \mathrm{~cm}^{-1}\right.$, $\left.\omega_{2 D, S i O 2,532}=2676.2 \mathrm{~cm}^{-1}\right){ }^{25}$ This blue-shift can be due to the interaction of graphene with the Au substrate ${ }^{26}$ and the annealing and cooling processes involved in AP-CVD that suggests the presence of residual compressive strain. ${ }^{27,28}$ As for coverage, large area micro-Raman maps of the integrated intensity $\left(I_{2 D}\right.$, Fig. $\left.1 \mathrm{C}\right)$, Raman shift $\left(\omega_{2 \mathrm{D}}\right.$, Fig. $\left.1 \mathrm{~d}\right)$, and spectral width $\left(\Gamma_{2 \mathrm{D}}\right.$, Fig. $\left.1 \mathrm{e}\right)$ extracted from single Voigt fitting of the $2 D$ Raman band showed that the monolayer graphene covered most of the substrate surface. Similar images from the $G$ and $D$ bands are shown in Supplementary Fig. 2. Cracks on the graphene, however, were also observed (Fig. 1c-e, enclosed by green lines), which can arise from the difference in thermal expansion coefficients between Au and graphene, especially since the substrate surface area is large (diameter $=10 \mathrm{~mm}$ ). Graphene is not mostly present within the crack resulting to no signal intensity from the $D, G$ and $2 D$ bands. Therefore, artefacts seen in the micro-Raman maps are due to fitting errors. The intense spot in Fig. $1 \mathrm{c}$ is a small island of multilayer graphene, which is a by-product of the AP-CVD process. From the Raman image (Fig. 1d), micro-domains (such as the blue areas) demarcated by wrinkles (such as the red dashed lines) are present throughout the graphene monolayer. The narrow wrinkles have relatively red-shifted Raman peaks $\left(\sim 2700 \mathrm{~cm}^{-1}\right)$ compared to the micro-domains $\left(\sim 2720 \mathrm{~cm}^{-1}\right)$ and indicate that the compressive strain experienced by the $\mathrm{Gr} / \mathrm{Au}(111)$ sample is relatively more relaxed at the wrinkles than at the micro-domains. We calculated the ratio of the integrated intensities of the $D$ and $G$ bands $\left(I_{D} / I_{G}\right.$, see Supplementary Fig. 3$)$. From the statistical data, majority of the areas except for the crack is defect-free and is selected for the following TERS measurements.

From topography measurements using our STM-TERS system, we found that the herringbone structure of the $A u(111)$ crystal was preserved (Fig. 1f) in multiple areas of the sample (see Supplementary Fig. 4), which is due to the attraction between graphene and $\mathrm{Au}^{29}$ Using a separate STM system, we also conducted atomic resolution topography and current imaging. From these images, the honeycomb lattice of graphene was observed, as shown in the current image in Fig. 1g (see Methods for experiment parameters). Similar images could be observed at various areas of the graphene sample (see Supplementary Fig. 5). Thus, further indicating that high quality monolayer graphene is coating the $\mathrm{Au}(111)$ surface without prominent defects.

\section{Subnanometre resolution STM-TERS in ambient}

Using our STM-TERS system (see Methods and Supplementary Fig. 6a), TERS measurements $(\lambda=632.8 \mathrm{~nm})$ shown in Fig. 2 were made in the area enclosed by the black box (Fig. 1c) situated far from the crack edge to ensure that there is no defect present. This area we call A1. Note that differences between Raman shift values of the $2 D$ band in Fig. $1 \mathrm{~d}$ and the subsequent Raman data in Fig. 2 is from the excitation wavelength dependence of the $2 D$ band (micro-Raman mapping $=532 \mathrm{~nm}$; TERS and far-field measurements $=632.8 \mathrm{~nm}){ }^{23}$ Appropriate incident laser powers were used to prevent any sample heating induced peak shift. Figure 2a shows representative Raman spectra for TERS on graphene (red line), tip retracted (black line), and TERS on a clean bare $\mathrm{Au}(111)$ substrate (blue line) in $\mathbf{A} 1$. The TERS spectrum from the $\mathrm{Gr} / \mathrm{Au}(111)$ sample has three distinct peaks, namely, $D\left(\omega_{D}=1334.3 \mathrm{~cm}^{-1}\right), G$ $\left(\omega_{\mathrm{G}}=1590.9 \mathrm{~cm}^{-1}\right)$, and $2 D\left(\omega_{2 D}=2656.6 \mathrm{~cm}^{-1}\right)$, along with a weak $D+D^{\prime}$ peak $\left(\omega_{D+D^{\prime}}=2921.1 \mathrm{~cm}^{-1}\right)$, which can be seen clearly upon averaging (Fig. $2 \mathrm{k}$ ) or with a longer exposure time 
a

Raman Shift $\left[\mathrm{cm}^{-1}\right]$

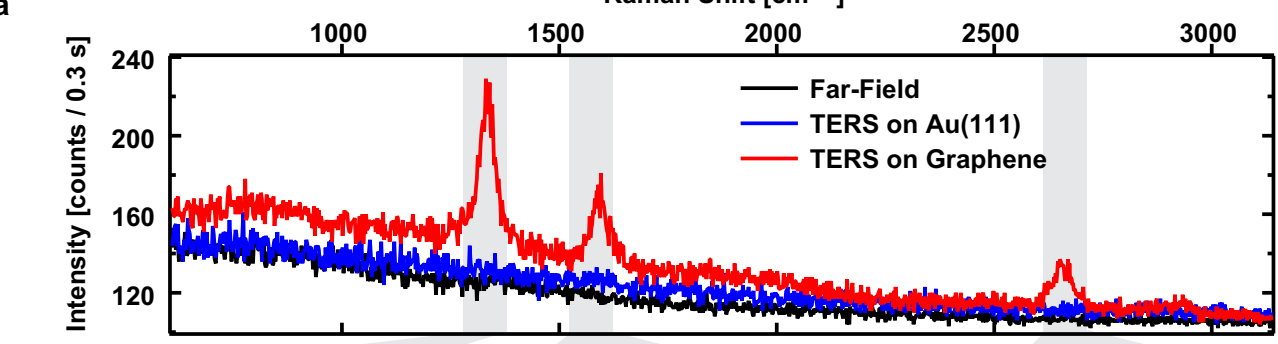

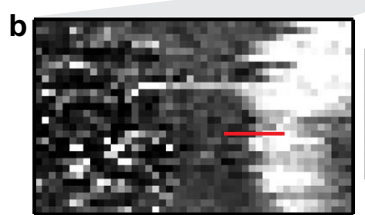
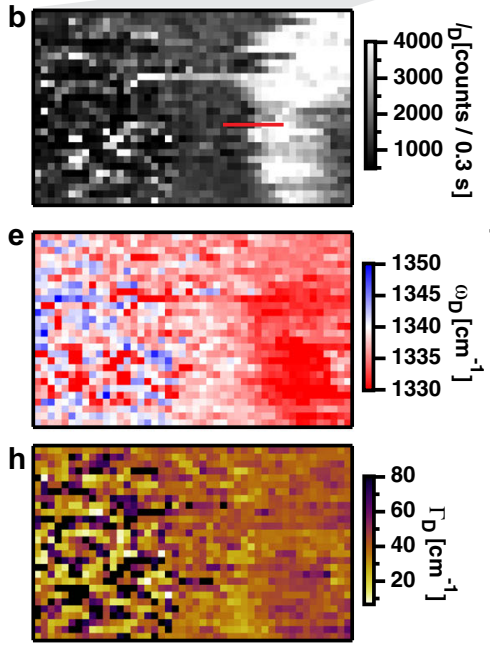
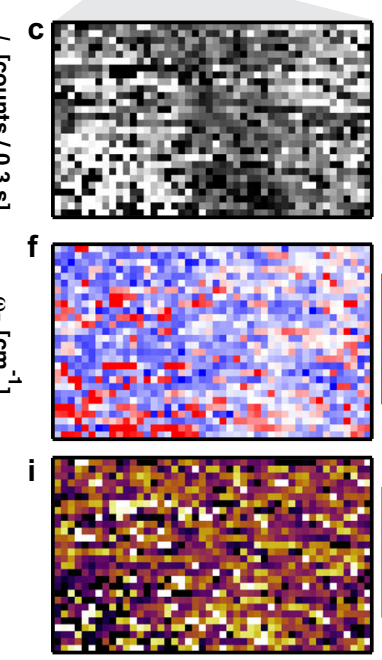

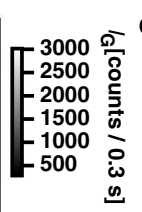

\section{d}
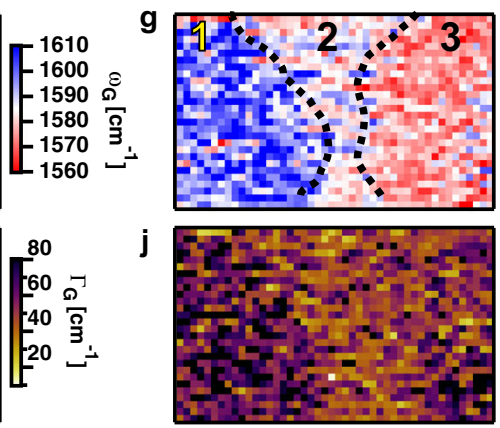

k

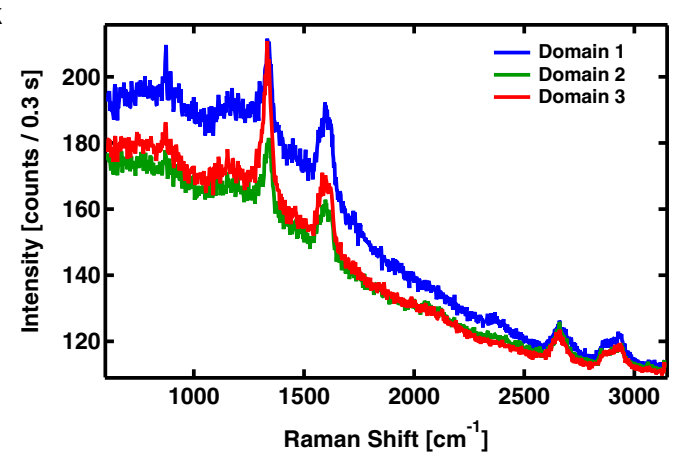

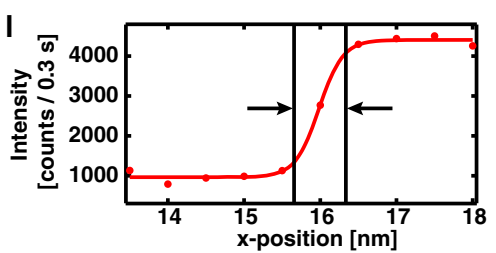

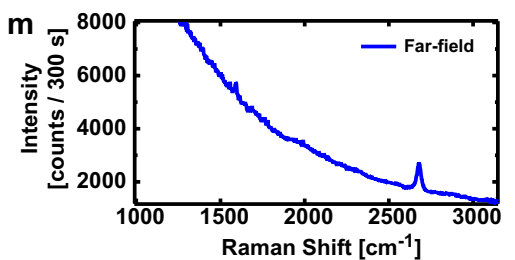

Fig. 2 STM-TERS spectroscopic imaging of area $A 1\left(23 \times 14 \mathrm{~nm}^{2}, 0.5 \mathrm{~nm} /\right.$ pixel) and spatial resolution. a Representative TERS on graphene (red), TERS on bare $A u(111)$ substrate (blue), and far-field (black, tip retracted) Raman spectra (exposure time $=0.3 \mathrm{~s})$. STM-TERS spectroscopic imaging of $D, G$ and $2 D$ bands showing integrated intensity: $\mathbf{b} D,\left(I_{D}\right), \mathbf{c} G,\left(I_{G}\right)$ and $\mathbf{d} 2 D,\left(I_{2 D}\right)$; Raman shifts: e $D,\left(\omega_{D}\right), \mathbf{f} G,\left(\omega_{G}\right)$ and $\mathbf{g} 2 D,\left(\omega_{2 D}\right)$, and spectral widths: $\mathbf{h} D,\left(\Gamma_{D}\right), \mathbf{i} G,\left(\Gamma_{G}\right)$ and $\mathbf{j} 2 D,\left(\Gamma_{2 D}\right)$. Red "x" mark in $\mathbf{d}$ is the point where TERS and far-field (tip retracted) spectra in Fig. $4 \mathrm{C}$ were measured. Three NDs with characteristic Raman peak shift are labelled in $\mathbf{g}$ as NDs 1, 2 and 3. Black lines in $\mathbf{g}$ are a guide to the eye to identify the extent of the NDs. Scale bar indicates $5 \mathrm{~nm}$. k Average TERS spectra from NDs 1 (blue), 2 (green) and 3 (red). I Line profile of step response taken at red line in $\mathbf{b}$. STM-TERS spatial resolution is $0.7 \mathrm{~nm}$ based on the $10-90 \%$ distance. $\mathbf{m}$ Long exposure (300 s) measurement of far-field Raman taken at the same point as TERS measurements showing only $G$ and $2 D$ bands. Excitation laser of $\lambda=632.8 \mathrm{~nm}$ with a laser power of $0.35 \mathrm{~mW}$ at the sample was used (tunnelling current: $40 \mathrm{pA}$; bias voltage: $300 \mathrm{mV}$ )

(see Fig. 4c). The $D^{\prime}$ peak $\left(\omega_{D}{ }^{\prime}=1620.7 \mathrm{~cm}^{-1}\right)$ is also present, however, its splitting from the $G$ peak cannot be clearly seen due to its low intensity. The peaks disappear when the tip is retracted (far-field), confirming that these peaks in the TERS spectrum are indeed enhanced Raman signals from the sample. The tip was also verified to be clean based on the featureless TERS spectrum taken on a bare $\mathrm{Au}(111)$ substrate. Hence, all detected Raman signals come from the graphene and not from any contaminants on the substrate surface. Full spectroscopic TERS imaging was conducted, simultaneously measuring the intensity $(I)$, Raman shift $(\omega)$, and spectral width ( $\Pi$ ) of the $D, G$ and $2 D$ bands (Fig. $2 \mathrm{~b}-\mathrm{j}$ ). Single Voigt fitting was used to extract the $D$ and $2 D$ bands, while double Voigt fitting was used for the $G$ band to exclude the contribution of the $D^{\prime}$ band. Taking the edge response of the line scan shown in Fig. 21 (taken from the red line in Fig. 2b), we found that our system achieved a spatial resolution of $0.7 \mathrm{~nm}$, which is currently the highest reported for STM-TERS in ambient. The calculated enhancement factor is $10^{10}$, based on the detected TERS and far-field (Fig. $2 \mathrm{~m}$ ) intensities, which were measured at the same point, and is comparable to the huge enhancement factor reported in gap-mode SERS. ${ }^{30}$ The enhancement is mainly due to the plasmonic nano-cavity and was not influenced by external 

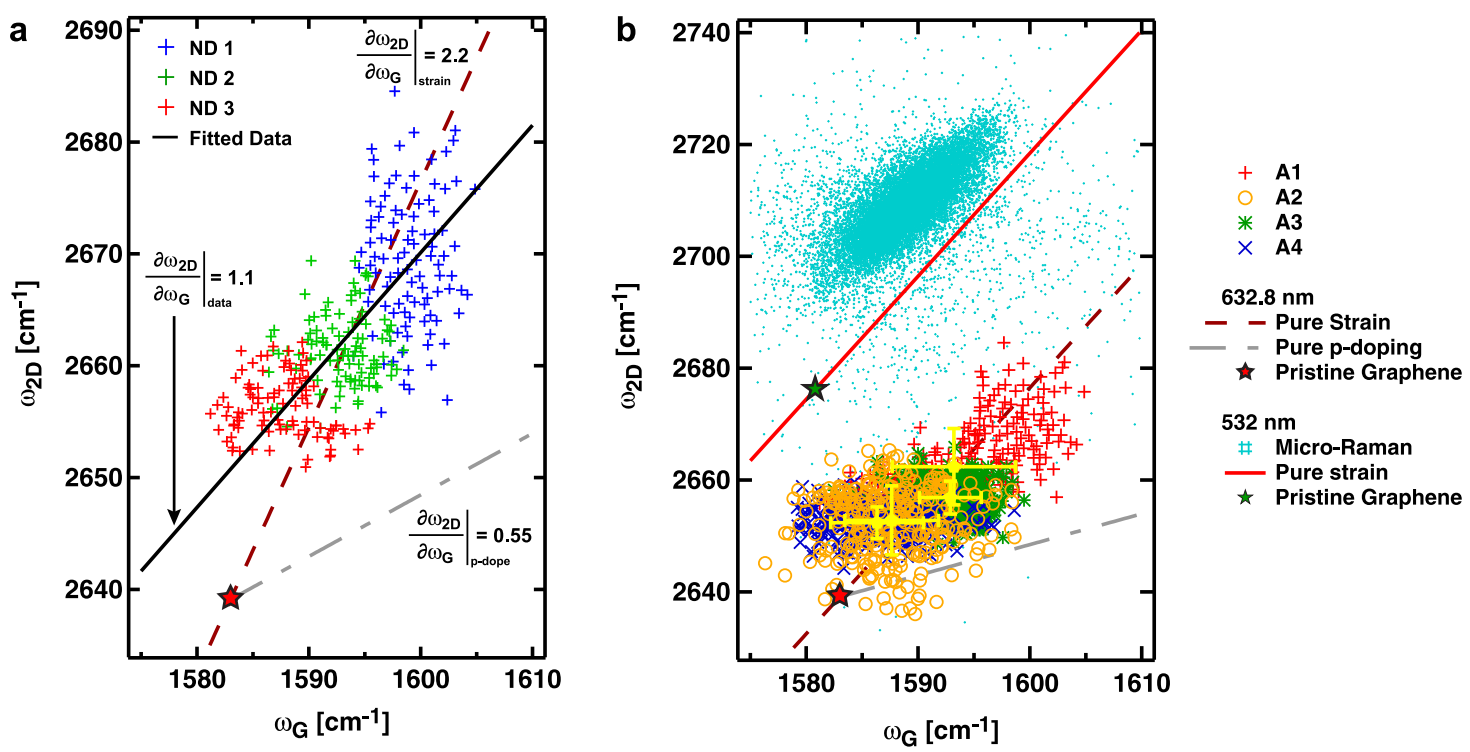

Fig. 3 Subnanometre strain analysis of $\mathrm{Gr} / \mathrm{Au}(111)$ from TERS data. a Correlation plot of $\omega_{2 D}$ Vs. $\omega_{G}$ of NDs 1 (blue), 2 (green) and 3 (red) found in Fig. $\mathbf{2 g}$ of $\mathbf{A} \mathbf{1}$. All data points of $\mathbf{A} \mathbf{1}$ are also plotted in $\mathbf{b}$ as red crosses. The red star represents the Raman frequencies for undoped and unstrained graphene on $\mathrm{SiO}_{2} / \mathrm{Si}$ substrate using $632.8 \mathrm{~nm}$ excitation ${ }^{35,37}$ and serves as the origin for the pure strain (consisting of biaxially isotropic strain and of biaxially anisotropic strain that less than $1 \%)^{28,38}$ and pure p-doping slopes. The maroon dashed line represents the expected Raman frequencies for pure strain with slope $\left(\partial \omega_{2 D} / \partial \omega_{G}\right)$ of $2.2^{28,38}$ while the grey dashed line is for pure $p$-doping with $\partial \omega_{2 D} / \partial \omega_{G}$ of $0.55 .^{34}$ The black solid line is the fitted data from NDs 1, 2 and 3 with $\partial \omega_{2 D} / \partial \omega_{G}$ of 1.1. b Correlation plot of $\omega_{2 D}$ Vs. $\omega_{G}$ of areas A1 (red cross), A2 (orange hollow circle), A3 (green asterisk) and A4 (blue $\times$ 's). Yellow hexagons represent average $\omega_{2 D}$ and $\omega_{G}$ frequencies for each area. Error bars indicate one standard deviation from the average. Correlation plot of $\omega_{2 D}$ vs. $\omega_{G}$ of micro-Raman data from Fig. 1 (green pound sign, excitation wavelength: $532 \mathrm{~nm}$ ). Green star indicates unstrained and undoped Raman peak of monolayer graphene on SiO $2 / \mathrm{Si}_{\text {substrate }}$ using $532 \mathrm{~nm}$ illumination $\left(\omega_{G, S i O 2,532}=1580.8 \mathrm{~cm}^{-1}, \omega_{2 D, S i O 2,532}=2676.2 \mathrm{~cm}^{-1}\right)$. Red line represents the expected Raman frequencies for pure strain at the 2.2 slope

factors such as the bias voltage introduced by the STM feedback system (see Supplementary Fig. 7). Subnanometre resolution and high signal enhancement were reproducible in our experiments owing to the high stability of our system (see Supplementary Fig. 8, data taken from another area called A2).

\section{Subnanometre resolution strain analysis}

We studied the subnanometre variations observed in the Raman peak images of $\mathbf{A} \mathbf{1}$ in Fig. 2 that can be attributed to the inhomogeneity of the interaction between graphene and the Au (111) substrate. From the Raman images (Fig. 2e-g), nanometresized domains or nano-domains (ND) as narrow as $5 \mathrm{~nm}$ in the $x$ direction can be discerned and are quite visible in the $\omega_{2 \mathrm{D}}$ image (Fig. $2 \mathrm{~g}$ ) of A1. These NDs within the micro-domain observed in Fig. 1d, e cannot be directly seen via micro-Raman measurements due to the averaging effect in the relatively larger focused spot. Three NDs labelled 1, 2 and 3 were observed in $\mathbf{A} 1$ with $\omega_{2 D}$ values decreasing from ND 1 to ND 3 . Figure $2 \mathrm{k}$ shows the average spectra from all three NDs. What is interesting to note is the D band is present in all areas regardless of the NDs as shown in the TERS data in Fig. 2b, k and the Supplementary Figs. 8 and 9, which is also an indication that the observed $D$ band does not originate from defects but is due to the plasmonic nano-cavity. This is because if we assume the $D$ band is from defects, then the entire scanned area should have defects and should exhibit a detectable micro-Raman signal. It is known that $\omega_{2 D}$ and $\omega_{G}$ are influenced by both strain ${ }^{21,31,32}$ and doping. ${ }^{33-35}$ There are works, however, indicating that the $G$ peak is more sensitive to doping ${ }^{34,36}$ whereas the $2 D$ peak is more sensitive to strain due to its higher Raman shift rate with respect to strain. ${ }^{32}$ To discern whether strain and/or doping is influencing $\omega_{2 D}$ and $\omega_{\mathrm{G}}$, Lee et al. ${ }^{28}$ optically separated the effects of strain and doping by constructing a correlation plot between $\omega_{2 D}$ and $\omega_{G}$ taken from micro-Raman data. We employed the same approach, but this time with subnanometric TERS data that is plotted in Fig. 3. In Fig. 3a, randomly chosen data points from each ND in Fig. $2 \mathrm{~g}$ are plotted and represented by crosses of different colours-blue from ND 1, green from ND 2 and red from ND 3. The red star indicates the deduced experimental microRaman peak values from ${ }^{35,37}$ for unstrained and undoped monolayer graphene on an $\mathrm{SiO}_{2} / \mathrm{Si}$ substrate illuminated by a $632.8 \mathrm{~nm}$ wavelength laser: $\left(\omega_{G}^{0}=1583 \mathrm{~cm}^{-1} ; \omega_{2 D}^{0}=2639.1 \mathrm{~cm}^{-1}\right)$. Dispersion of the 2D band at $100 \mathrm{~cm}^{-1} \mathrm{eV}^{-1}{ }^{37}$ was considered. The maroon dashed line with a slope $\left(\partial \omega_{2 D} / \partial \omega_{G}\right)$ of 2.2 corresponds to the rate of change of $\omega_{2 D}$ and $\omega_{G}$ under pure strain. The definition of pure strain in this case is a combination of both biaxially isotropic and biaxially anisotropic (generally called "biaxial" and "uniaxial", respectively) strain below $1 \% .{ }^{28,38}$ The grey dashed line with $\partial \omega_{2 D} / \partial \omega_{G}$ of 0.55 corresponds to pure pdoping. ${ }^{34}$ Compared to $\left(\omega_{G}^{0}, \omega_{2 D}^{0}\right)$, blue-shifting of the Raman modes is observed, which implies compressive strain is present in all three NDs. Collectively, the NDs exhibit a linear trend with $\partial \omega_{2 D} / \partial \omega_{G}$ of $1.1 \pm 0.1$ (black line, Fig. 3a). This slope deviates from the pure strain slope of 2.2, meaning both strain and slight $p$ doping are influencing the detected shift of the Raman peaks. These observations are attributed to two factors: (1) the cooling process during AP-CVD and (2) electron charge transfer between graphene and the $\mathrm{Au}(111)$ substrate. The cooling process after graphene synthesis in AP-CVD induces strain due to the different thermal expansion coefficients of graphene and Au. In our case, the strain is compressive in nature, which is similar to the findings of Oznuluer et al. ${ }^{27}$ where they grew graphene on a polycrystalline Au partially (111) oriented surface via CVD. As for the slight p-type doping in the probed areas, this can be caused by electron charge transfer from graphene to the Au(111) surface, which has been both experimentally and theoretically demonstrated in literature. ${ }^{26,39-41}$ 

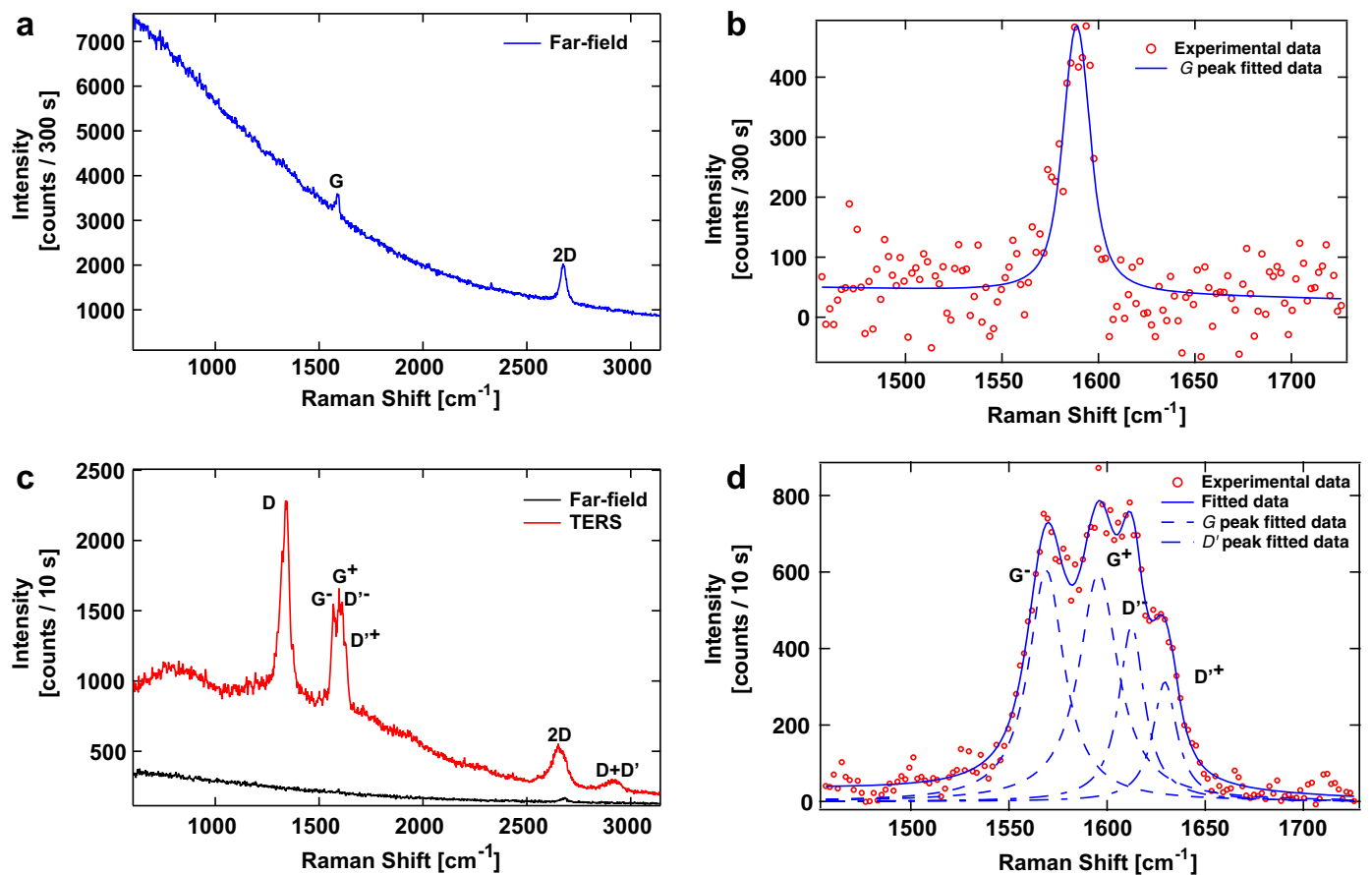

Fig. $4 \mathrm{G}$ band and $\mathrm{D}^{\prime}$ band splitting due to biaxially anisotropic strain. a Far-field spectrum measured with $300 \mathrm{~s}$ exposure time showing single $\mathrm{G}$ and $2 \mathrm{D}$ bands. This spectrum was taken at the same point where TERS spectra was measured to serve as a reference spectrum for enhancement calculations. b Single Raman peak $G$ band from a measured in the far-field and fitted with a single Voigt. c Representative TERS (red line) and far-field (black) spectra measured with $10 \mathrm{~s}$ exposure time at point $\mathbf{x}$ in Fig. $2 \mathrm{~d}$. $\mathbf{d}$ Multiple Raman peaks from $\mathbf{c}$ consisting of $G^{-}$, $G^{+}, D^{\prime-}$ and $D^{\prime+}$ bands measured using TERS (tunnelling current: $40 \mathrm{pA}$; bias voltage: $300 \mathrm{mV}$ ) and fitted with a quadruple Voigt. Background has been subtracted in $\mathbf{b}$, $\mathbf{d}$. During the experiments, an excitation laser wavelength of $632.8 \mathrm{~nm}$ with a laser power of $0.35 \mathrm{~mW}$ at the sample was used

\section{Anisotropy in subnanometric strain}

We scanned other areas of the $\mathrm{Gr} / \mathrm{Au}(111)$ sample where we also observed NDs and subnanometre variations (see Supplementary Figs. 8 and 9) from their TERS images. These areas we call A2, A3, and A4. We again plotted random data points from A1 (red crosses, same data points from Fig. 3a), A2 (orange hollow circles), A3 (green asterisks), and A4 (blue $x^{\prime} s$ ) into a single correlation plot as shown in Fig. 3b. The yellow hexagons are the average $\omega_{2 D}$ and $\omega_{G}$ of each area and the yellow error bars show the measure of one standard deviation. The variation of $\omega_{2 D}$ and $\omega_{G}$ in all areas is quite large, but the points generally scatter along the 2.2 strain slope, which clearly suggests that the comparison of all the data points in each area exhibits the similar effect of averaging in micro-Raman measurements. Figure $3 \mathrm{~b}$ shows the comparison between the micro-Raman measurements from Fig. 1 and the TERS data. Although different laser wavelengths were used (microRaman $=532 \mathrm{~nm}$; TERS $=632.8 \mathrm{~nm}$ ), the behaviour of the peaks can still be compared. It can be seen that most of the microRaman data does not fall on the 2.2 strain slope unlike the TERS data. The upshift of the micro-Raman data can be attributed to the $\pi$ orbital of graphene that is hybridised with the $d$ orbital of the Au (111) substrate ( $\pi-d$ hybridisation). ${ }^{42}$ Such upshift has been observed before in other substrates such as hexagonal boron nitride. ${ }^{20}$ In contrast to micro-Raman, not only substrate-graphene interaction but also tip-graphene interaction affects the observed TERS signal, especially when a highly confined light source (focus spot diameter $\sim 1 \mathrm{~nm}$ ), such as the TERS tip, is introduced to the system. One possible explanation for the tip-graphene interaction, that needs further verification, is the phonon confinement in TERS due to the highly confined light source (spot diameter $\sim 1 \mathrm{~nm}$ ) at tip end. Our idea is based on the work of Arora, et al. ${ }^{43}$ on phonon confinement in nanostructured materials. Their claim is that illuminated light gets confined within a nanostructure, whose size is less than $10 \mathrm{~nm}$, when this nanostructure is located in a medium that cannot propagate the scattered light. The isolation of the particle causes a relaxation of the zone-centre optical phonon selection rules. As a result, the detected Raman signal from that sample exhibits both a shift in the Raman peak and asymmetric broadening. In the case of TERS, we have the inverse. Instead of a nano-sized structure illuminated with diffraction-limited light, we have a large area 2D nanomaterial (that can be assumed to be semi-infinite) illuminated with the highly confined light source. Although the generated phonon can propagate within the 2D nanomaterial, due to the confined light source with a larger electric field gradient, the probed area is still limited and the phonons of $k \neq 0$ can be generated. The magnitude of the shift and the broadening of the peak is dependent on the dispersion curve of the material. ${ }^{43}$

In micro-Raman measurements, nanometre scale strain variations that limit the carrier mobility of graphene ${ }^{20,37,44}$ are indirectly observed through the broadening of the $2 D$ peak spectral width due to the diffraction-limited spatial resolution. As a consequence, the nature of these strain variations cannot be determined. This is not a problem, however, when using our STMTERS system. The nature of the strain is evident when we compare the far-field spectrum measured via micro-Raman and the TERS spectrum from A1 in Fig. 4. The micro-Raman measurement (Fig. 4a) shows a single $G$ peak $\left(\omega_{G}=1588.7 \mathrm{~cm}^{-1}\right.$, Fig. $\left.4 \mathrm{~b}\right)$ and a single $2 D$ peak $\left(\omega_{2 D}=2675.4 \mathrm{~cm}^{-1}\right)$ that implies biaxially isotropic strain is present, since all symmetries are preserved and no splitting of the $G$ and the $2 D$ bands are observed. ${ }^{45}$ This biaxially isotropic strain is compressive, as discussed earlier, since the observed Raman peaks are blue-shifted with respect to unstrained and undoped graphene on an $\mathrm{SiO}_{2} / \mathrm{Si}$ substrate $\left(\omega_{G}^{0}, \omega_{2 D}^{0}\right)$. It is possible though that not all areas within the laser-focused spot are biaxial isotropically strained, but due to the averaging effect, this information is lost. Such information is re-obtained through 
TERS measurements. In the TERS spectrum (Fig. 4c) taken from point $\mathbf{x}$ in Fig. $2 d$, a clear splitting of the $G$ band into the $G^{-}\left(\omega_{G}{ }^{-}\right.$ $\left.=1569.1 \mathrm{~cm}^{-1}\right)$ and $G^{+}\left(\omega_{G}^{+}=1596.2 \mathrm{~cm}^{-1}\right)$ bands is observed, which denotes that biaxially anisotropic strain is present. The $G$ band originates from the $E_{2 g}$ mode which is doubly degenerate because of graphene's hexagonal lattice symmetry. ${ }^{46}$ This degeneracy is lifted when strain is present and, as a result, $G$ band splitting can be observed. The $G^{-}$band corresponds to the modes parallel to the applied strain and the $G^{+}$band corresponds to the modes perpendicular to the applied strain. ${ }^{21,31,47}$ Therefore, the $G^{-}$Raman peak position tends to be more shifted compared to the $G^{+}$band with respect to the degenerate $G$ band of unstrained graphene. As for the $2 D$ band $\left(\omega_{2 D}=2655.8 \mathrm{~cm}^{-1}\right)$, spectral width broadening is observed, which can be due to the presence of the $2 D^{-}$and $2 D^{+}$peaks, however, splitting is not evident. The mechanism behind $2 D$ band splitting is not as straight forward as $G$ band splitting and is a combination of various factors, namely, strain, polarisation of the incident light with respect to the applied strain, and the phonons involved in the double resonance (DR) process. For an in-depth discussion on $2 D$ band splitting, we refer the reader to Ref. ${ }^{48}$

From the TERS data in Fig. 4c, we calculated the amount of biaxially anisotropic strain based on the $2 D$ band, since the absolute shift of the $G$ band is affected not only by strain but also from doping. Biaxially anisotropic strain is defined as $\mathrm{s}^{21,49}$

$$
\varepsilon=\frac{\Delta \omega_{2 D}}{\omega_{2 D}^{0}(1-\nu) \gamma_{2 D}^{u n i}},
$$

where $\varepsilon$ is the strain along the longitudinal direction $\left(\varepsilon=\varepsilon_{l l}\right), \omega_{2 D}^{0}$ is the Raman shift of the $2 D$ peak from undoped and unstrained graphene on $\mathrm{SiO}_{2} / \mathrm{Si}, \Delta \omega_{2 \mathrm{D}}$ is the difference of the experimentally measured $2 D$ band Raman shift from our $\mathrm{Gr} / \mathrm{Au}(111)$ sample with respect to $\omega_{2 D}^{0}, v$ is Poisson's ratio of $A u(v=0.42),{ }^{49}$ and $\gamma_{2 D}^{u n i}$ is the biaxially anisotropic Grüneisen parameter for the $2 D$ peak $\left(\gamma_{2 D}^{\text {uni }}=2.7\right) .^{5}$ The calculated $\varepsilon$ is $-0.4 \%$, whose negative sign indicates that compressive strain is present and agrees with the expected compressive strain in our $\mathrm{Gr} / \mathrm{Au}(111)$ sample as discussed in the previous Subnanometre Resolution Strain Analysis Subsection. A point of contention on the calculated compressive strain is the observed $G^{-}$and $G^{+}$Raman shifts. When compressive strain is present, $\omega_{G}{ }^{-}>\omega_{G}{ }^{+}$is expected whereas our TERS data on the $G^{-}$and $G^{+}$bands shows the opposite, $\omega_{G}{ }^{-}<$ $\omega_{G}{ }^{+}$, a trend that is generally exhibited when tensile strain is present. The TERS data on graphene in literature that we have come across show only single $G$ bands. Therefore, it is difficult to draw some point of comparison. Some other local property of graphene may be contributing to the shift of the split $G$ band, such as doping. ${ }^{34,36}$ However, the calculated amount of doping (carrier concentration) at point $\mathbf{x}$ is much higher compared to areas A1, A2, A3 and A4 (see Supplementary Information Section 8). This indicates that doping alone cannot induce such a large shift. Aside from doping, another possible reason for the $G^{-}$and $G^{+}$Raman shifts is the high photon confinement of the plasmonic nano-cavity used to excite the sample. This idea we expound on in the Discussion Section of this paper. Further perusing the $G$ band in Fig. $4 \mathrm{c}$, the splitting of the $D^{\prime}$ band into the $D^{\prime}-\left(\omega_{\mathrm{D}^{\prime}}{ }^{-}=\right.$ $\left.1612.7 \mathrm{~cm}^{-1}\right)$ and $D^{\prime+}\left(\omega_{D^{\prime}}{ }^{+}=1629.1 \mathrm{~cm}^{-1}\right)$ is evident as shown in Fig. $4 \mathrm{~d}$. The peak position assignments are roughly based on $G$ peak splitting. The effect of strain on the $D^{\prime}$ peak was initially reported by Mohiuddin et al., ${ }^{21}$ however, they were not able to experimentally observe the split $D^{\prime}$ peaks via micro-Raman due to the $D^{\prime}$ band's weak intensity. To the best of our knowledge, no other group has reported the experimental observation of $D^{\prime}$ peak splitting using TERS, which shows the advantage of the plasmonic nano-cavity and its activation of forbidden phonon modes in TERS over micro-Raman.

\section{DISCUSSION}

High photon confinement and a well-defined sample are central to obtain high spatial resolution. An important evidence of the strongly confined electric field beneath the tip apex is our observation of the forbidden $D, D^{\prime}$ and $D+D^{\prime}$ phonon modes (Fig. 4c). These modes are forbidden under far-field excitation for defect-free graphene, since there are no defects present to preserve the momentum conservation by electron-phonon scattering during DR (Fig. 5a). In the near-field excitation of defect-free graphene, momentum conservation is preserved by the large $k$ vectors that make non-vertical optical transitions possible. ${ }^{50}$ This process is the $\mathrm{FGE}^{17}$ or the plasmon-mediated process. ${ }^{16}$ Large $k$ vectors are present because of the nanoconfined field with a diameter $\sim 1 \mathrm{~nm}$ found in the gap between the tip apex and the $\mathrm{Gr} / \mathrm{Au}(111)$ sample resulting to wavenumbers as large as $k_{N F} \approx p / d \approx 10^{7} \mathrm{~cm}^{-1}$. This value is comparable to the wavevector $q$ of the full phonon dispersion curve, reaching up to the boundary of the Brillouin zone that has a value of $p / a \sim 10^{8}$ $\mathrm{cm}^{-1}$ where $a$ is the lattice parameter. Hence, the momentum is conserved not only for the $D$ and $D^{\prime}$ phonon mode, but even the $D+D^{\prime}$ combination phonon mode, making all three forbidden modes detectable. The vertical transition during excitation is also forbidden due to the high photon confinement of the plasmonic nano-cavity. ${ }^{51}$ Based on these facts, Fig. $5 b$ presents one of the possible pathways to excite and detect the $D$ and the $D+D^{\prime}$ modes considering all non-vertical transitions.

From the perspective of Raman scattering, the $D$ peak originates from phonons whose wavevectors are close to the $K$ and $K^{\prime}$ points. These modes have $A_{1}$ and $B_{1}$ symmetry as shown in Fig. $5 c^{52}$ If the size of the confined illuminating electric field is comparable to the unit cell of the $D$ phonon, the $D$ phonon modes can be excited, resulting to the detection of the $D$ Raman peak, which is the case in our TERS experiments. Compared to other TERS works on defect-free graphene, ${ }^{16}$ the intensity of the detected $D$ band is higher since a component of the incident electric field is parallel to the graphene sample due to the tilted tip (see Methods and Supplementary Fig. 10). This configuration is advantageous in studying graphene since its Raman peaks come from in-plane vibrations. ${ }^{47}$ Aside from high photon confinement, subnanometre resolution and high signal enhancement were possible because of (1) high reproducible sharp Au tips for better phonon confinement; ${ }^{53}$ (2) high stability via an improved all-metallic environment control chamber; and (3) a highly defined atomically flat sample. With this high spatial resolution, the local properties of our $\mathrm{Gr} / \mathrm{Au}$ (111) sample, such as strain, were studied with subnanometre precision.

Our data show that subnanometre strain analysis using TERS data is possible and even $D^{\prime}$ splitting can be experimentally observed. It should be noted though that the TERS spectrum in Fig. 4c was taken with an exposure time of $10 \mathrm{~s}$, which is why splitting could be clearly seen. In contrast to single point analysis, assessing the strain in the entire area is challenging for now, since during TERS imaging, a relatively short exposure time of $0.3 \mathrm{~s}$ per point is practically used due to the limited stability resulting to limited signal-to-noise ratios that are not strong enough to discern the splitting of the peaks. Hence, all the peaks during TERS imaging appear to be single peaks - making biaxially isotropic or anisotropic strain indistinguishable.

Up to this point, most of our discussion has focused on how the defect-related Raman bands, $D, D^{\prime}$ and $D+D^{\prime}$ are affected by the high photon confinement of the plasmonic nano-cavity. We now look at the $G$ band and its consequence to near-field strain analysis. Here, we present our hypothesis that could be verified in future works. The wave vector of the $\mathrm{G}$ phonon mode is $q=0$, which is the $\Gamma$ point. When $q \neq 0$, this doubly degenerate phonon, as shown in the phonon dispersion relations, splits into the iTO and LO branches. ${ }^{54}$ These split phonon modes not located at the 
a
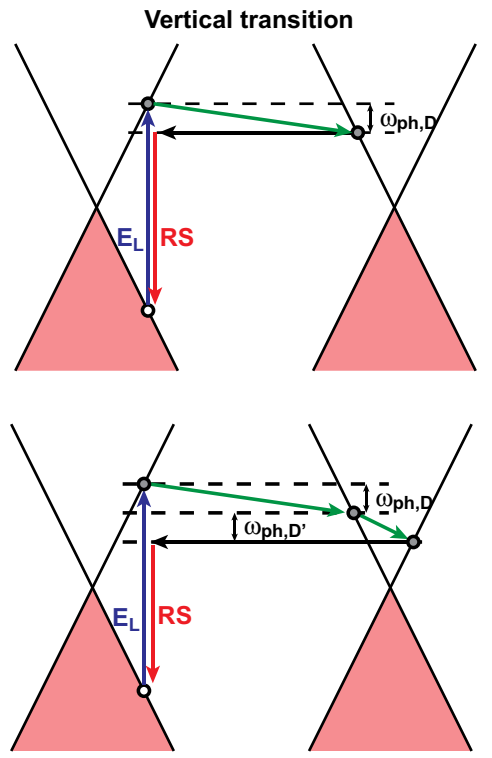

C

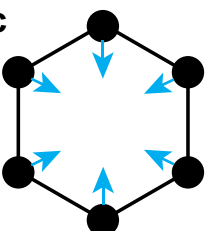

b
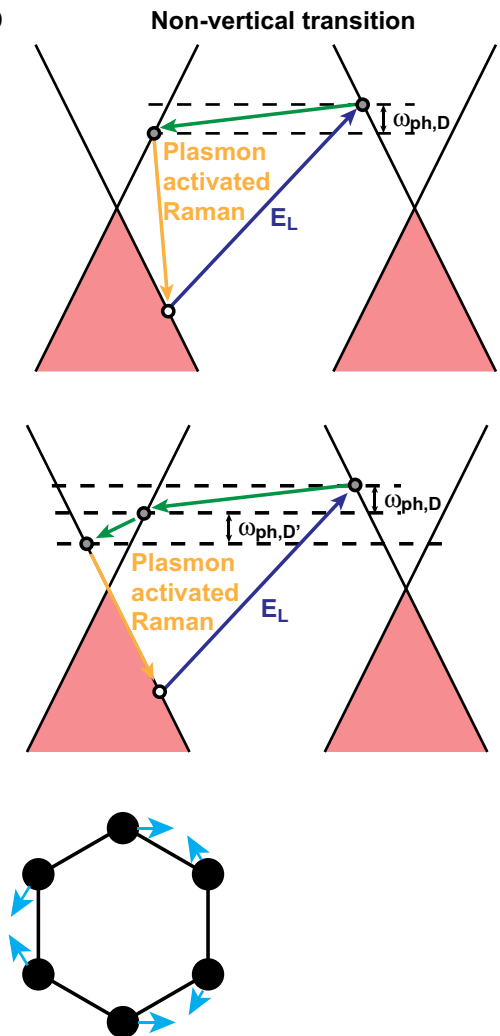

Fig. 5 Mechanisms of activation of $D$ and $D+D^{\prime}$ bands. For the activation of the $D$ and $D+D^{\prime}$ bands, in a when laser light excites the graphene sample, vertical interband transition with photon absorption ( $E_{L}$, blue line) occurs and is followed by phonon emission by electronphonon scattering (green line). When a defect is present, it scatters the electron with exchanged momentum (far-field, black line) and a photon is emitted as Raman scattering after electron-hole recombination (red line). This process is called double resonance. ${ }^{23} D+D^{\prime}$ follows a similar process but electron-phonon scattering occurs twice (green lines) before being scattered by a defect (far-field, black line). $\mathbf{b}$ When no defect is present and graphene is illuminated by the TERS tip, non-vertical transitions occur during excitation due to high photon confinement and also after phonon emission via a plasmon-mediated process (near-field, orange line). Activation of the $D^{\prime}$ band can also be explained in the same manner. c Local lattice vibrations of $A_{1}$ (left) and $B_{1}$ (right) symmetry that are the source of $D$ Raman peak

$\Gamma$ point are not allowed to be observed during far-field measurement, whereas it should be possible to excite these split phonon modes through the high photon confinement of the plasmonic nano-cavity. The mechanism behind this excitation lies on the non-vertical optical transition similar to the activation of the forbidden modes, such as the $D, D^{\prime}$ and $D+D^{\prime}$ bands. ${ }^{51}$ This unique process induced in near-field excitation $(q \neq 0)$ different from the far-field Raman selection rule $(q=0)$ would make an interesting turn in strain analysis, particularly for uniaxial strain where peak splitting of the $G$ band due to a broken lattice symmetry occurs. Peak splitting by uniaxial strain could be coupled with peak splitting by the confined field resulting to Raman shifts that may not be predicted using the current set of theories based on many systematic far-field Raman experiments. One example would be the $G^{-}$and $G^{+}$Raman peak positions stated in the Anisotropy in Subnanometric Strain Section of this paper, wherein the split peak positions indicate tensile strain contrary to the calculated and overall observed compressive strain in the graphene sample. Furthermore, the difference between the Raman peaks $\left(\Delta \omega_{G^{ \pm}}=\omega_{G^{+}}-\omega_{G^{-}}\right)$is $27.1 \mathrm{~cm}^{-1}$. Theoretically, such a large difference would indicate a strain level $(\varepsilon)$ of $\sim 1.2 \%$ and this value is much larger than the computed $-0.4 \%$ based on the $2 \mathrm{D}$ band. For the case of $-0.4 \%$ strain, the theoretical amount of $\Delta \omega_{G^{ \pm}}$would be $\sim 9 \mathrm{~cm}^{-1}$. This masking of the actual strainrelated shift can also be the reason why the $2 D$ band does not show clear splitting as compared to the $G$ band, since the actual strain may not be high enough to see clear splitting for both peaks. As of now, it would be difficult to surmise if the additional
$18.1 \mathrm{~cm}^{-1}$ shift of $\Delta \omega_{G^{ \pm}}$is purely due to near-field induced splitting, but it is necessary to explore such kind of contribution and develop more theories that address near-field excitation especially since technology is rapidly moving to the nanometre regime and high resolution techniques such as TERS will be necessary for materials characterisation.

In conclusion, STM-TERS in ambient has now reached subnanometre resolution and, with the development of more methods for near-field analysis, will be useful in determining the as-fabricated properties of devices, such as strain. With the high photon confinement and FGE provided by the plasmonic nano-cavity, we have gained access to the full phonon spectrum to probe the properties of defect-free materials. Now, the bigger question is what additional information would these previously forbidden phonon modes provide as compared to the allowed modes. These questions and ideas are not only applicable to graphene but also to various members of the $2 \mathrm{D}$ nanomaterial family.

\section{METHODS}

Micro-Raman measurements

Large area micro-Raman measurements were conducted using Raman 11 (Nanophoton) with $532 \mathrm{~nm}$ laser excitation wavelength and an NA $=0.9$ objective lens $(\times 100$, Nikon). Line illumination was used for fast data acquisition during imaging. Laser power was fixed below $1 \mathrm{~mW}$ at the sample to prevent sample heating. An exposure time of $30 \mathrm{~s}$ per line was used. Voigt fitting was used to extract the $D, G$ and $2 D$ bands. A constant Gaussian component (FWHM) of $8.5 \mathrm{~cm}^{-1}$ was incorporated in the fitting 
to take into consideration the contribution of the spectrometer to the signal linewidth.

\section{Sample and tip preparation}

The substrate is an $\mathrm{Au}(111)$ single crystal disk (MaTeck $\mathrm{GmbH}$ ) with a diameter of $10 \mathrm{~mm}$ and thickness of $2 \mathrm{~mm}$. It was immersed in piranha solution $\left(\mathrm{H}_{2} \mathrm{SO}_{4}: \mathrm{H}_{2} \mathrm{O}_{2}=3: 1\right)$ for 30 minutes to remove any contaminants and then flame annealed to ensure that the surface was atomically flat. Monolayer graphene was grown directly on the substrate via AP-CVD. We implemented a similar method used in Ref. ${ }^{22}$ and present a brief description of the AP-CVD process. The graphene was grown in a quartz furnace having a diameter of $15 \mathrm{~mm}$. To begin the growth process, the clean and atomically flat $\mathrm{Au}(111)$ substrate was placed into the quartz furnace and initially exposed to $\operatorname{Ar}(99.999 \%)$ gas that was flowing into the furnace at a rate of $500 \mathrm{sccm}$ (cubic centimetre per minute at standard temperature and pressure: $0^{\circ} \mathrm{C}, 1 \mathrm{~atm}$ ) for $60 \mathrm{~min}$ while being heated at $1025^{\circ} \mathrm{C}$. This temperature was maintained throughout the entire AP-CVD process. Afterwards, graphene was grown on the $\mathrm{Au}(111)$ surface by introducing a gas mixture composed of $1 \mathrm{sccm}$ of $\mathrm{CH}_{4}(99.99 \%)$ and $750 \mathrm{sccm}$ of $\mathrm{Ar}$ (99.999\%) for $6 \mathrm{~min}$. The $\mathrm{CH}_{4}$ gas was the carbon source to form graphene. After growth, the graphene on $\mathrm{Au}(111)$ sample was annealed for 30 min while $\operatorname{Ar}(99.99 \%)$ was flowing at $300 \mathrm{sccm}$. After APCVD, the sample was further annealed at $300^{\circ} \mathrm{C}$ for $2 \mathrm{~h}$ to remove any carbon-based contaminants on the surface of the $\mathrm{Gr} / \mathrm{Au}(111)$ sample. The tip was prepared via electrochemical etching of an Au wire (diameter = $0.25 \mathrm{~mm}$ ) using a three-electrode system in potassium chloride. ${ }^{53}$ The fabricated Au tip has a diameter of $32 \mathrm{~nm}$ and is shown in Supplementary Fig. 10a. Through this method, highly reproducible sharp Au tips were made and used for the TERS experiments. The etching protocol is found in Ref. ${ }^{53}$

Simulations for using oblique illumination on a tilted tip to excite the in-plane modes of graphene via TERS

To efficiently excite the Raman peaks in graphene that originate from inplane vibrational modes, ${ }^{47}$ a strong electric field parallel to the graphene surface must be present. In TERS, however, it is the electric field that is parallel to the metal tip that is intense and this tip is typically perpendicular to the sample. As a result, the enhanced electric field at the tip apex cannot couple well with the in-plane vibrational modes of graphene. One solution to this problem is to obliquely introduce laser light onto a tilted tip. Finite-difference time-domain (FDTD) simulations were conducted using commercially available software (Lumerical) to determine the field distribution and strength of the electric field parallel to the surface of the substrate $\left(\mathbf{E}_{\mathbf{X}}\right)$ when using a laser illumination wavelength of $632.8 \mathrm{~nm}$. The model for the simulations is shown in Supplementary Fig. 10b. The simulation parameters are as follows. An Au tip with a radius $r=$ $16 \mathrm{~nm}$ and cone angle $a=5^{\circ}$ was positioned a distance $d=1 \mathrm{~nm}$ away from an Au substrate. The tip was tilted at an angle $\beta=27^{\circ}$ from the substrate normal. A tip length of $L=500 \mathrm{~nm}$ was used to reduce computation time. This tip-substrate system was illuminated by a linearly polarised electric field $(\lambda=632.8 \mathrm{~nm})$ at an incident angle of $\theta_{i}=60^{\circ}$. Polarisation was oriented along the $z$-axis of the tip (p-polarised). These parameters were based on the actual Au tip used in the TERS experiments (Supplementary Fig. 10a). The FDTD mesh size was $x=y=0.5 \mathrm{~nm}$ per pixel and $z=0.25 \mathrm{~nm}$ per pixel. Higher resolution was used in the $z$-component to clearly resolve the electric field distribution at the gap. Supplementary Fig. 10c-d show the simulated $x$ - and $z$-components of the enhanced electric field at the gap between the tilted tip and substrate, respectively. The $x$-component of the field is weaker than the $z$-component, which is expected since it is the longitudinal component of the incoming $p$ polarised field that efficiently couples with the tip. ${ }^{47}$ But from the simulations we found that the intensity of $\mathbf{E}_{\mathbf{X}}$ for the tilted tip is higher than the non-tilted tip (Supplementary Fig. 10e). This increase in intensity can be due to the additional $\mathbf{E}_{\mathbf{X}}$ component that is projected onto the substrate when the tip is tilted. From the simulation results, we deduced that it is feasible to excite and detect the Raman signals from our $\mathrm{Gr} / \mathrm{Au}$ (111) sample during the STM-TERS experiments.

\section{High stability STM-TERS system in ambient}

For the TERS experiments, we used our STM-TERS system in ambient (Supplementary Fig. 6a) based on our previous system explained in ${ }^{13}$ but incorporated major improvements for better system stability. These improvements played an important role in helping us attain subnanometre resolution in ambient conditions. The entire system is housed in an allmetal chamber that can be sealed with a metal top door. The purpose of this all-metal chamber is four-fold: (1) it effectively shields the system from unwanted vibrational and mechanical noise in the immediate environment; (2) a completely dark environment free of stray light can be attained; (3) a controlled environment in terms of temperature and humidity can be maintained; and (4) the chamber can be purged with nitrogen gas, thus, dramatically reducing the humidity in both the chamber and the experiment system. Supplementary Fig. $6 \mathrm{~b}-\mathrm{d}$ show the temperature and the humidity performance inside and outside the chamber and also after nitrogen gas purging, respectively. The reduction of humidity is particularly useful in our experiments wherein we use an Au tip to probe the sample. $\mathrm{Au}$ is known to be hydrophilic and any water molecule lodged on the Au tip apex will be detrimental to the STM-TERS experiments.

\section{STM topography measurements}

Herringbone structure STM topography measurements were made using our STM-TERS system. The tips used were Au tips that were modified via pulsing. A tunnelling current of $0.5 \mathrm{nA}$ and a bias voltage of $300 \mathrm{mV}$ were used during topography measurements. These parameters were also used for Supplementary Fig. 4. It should be mentioned here that for the TERS results, no topography image is shown. This is due to the nature of the electrochemically etched Au tips ${ }^{53}$ that were used for both STM imaging and TERS. In order to obtain clear STM topography images of the herringbone structure, modification of the Au tip is necessary by pulsing electricity through the tip but at the cost of losing tip enhancement due to the change in the cone shape of the tip. This is one of the remaining issues when conducting TERS experiments in ambient.

The atomic resolution STM image was taken using a separate STM system operating in ambient conditions (Agilent 5500 SPM, Agilent Technologies). The image was taken at constant current mode using a Pt/lr tip with a tunnelling current of $2 \mathrm{nA}$ and a bias voltage of $5 \mathrm{mV}$.

\section{TERS measurements}

The schematic of our experimental setup is shown in Supplementary Fig. 6a. The tip is tilted $27^{\circ}$ from the surface normal of the sample for a larger solid angle. Linearly polarised light parallel to the tip $(\lambda=632.8 \mathrm{~nm}, \mathrm{He}-\mathrm{Ne})$ illuminates the sample with an angle of incidence, $\theta_{i}$, of $60^{\circ}$. This laser light is focused onto the sample by a long working distance objective lens (NA $=0.6, \times 50$, Nikon) with a focus spot diameter of $2.6 \mu \mathrm{m}$ based on the long axis of the ellipse. Reflected light is collected by the same objective lens and detected by the liquid nitrogen cooled CCD of the spectrometer (grating $=600 \mathrm{~g} / \mathrm{mm}$, focal length $=30 \mathrm{~cm}$, slit width $=100 \mu \mathrm{m}$ ). A multimode fibre $(\mathrm{NA}=0.12$, core diameter $=105 \mu \mathrm{m})$ was used for efficient photon detection. Laser power of $\sim 0.35 \mathrm{~mW}$ at the sample was used to prevent sample heating. For the STM-TERS experiments, the bias voltage was $300 \mathrm{mV}$ and the tunnelling current was kept at $40 \mathrm{pA}$. To remove the contribution of the spectrometer, a Voigt line shape was used for fitting with a constant Gaussian component having an FWHM of $9.7 \mathrm{~cm}^{-1}$. Only the Lorentzian component was extracted and used to visualise the data. Single Voigt fitting was used for $D$ and $2 D$ band images, while double Voigt fitting was used for $G$ band images to exclude the contribution of the $D^{\prime}$ band.

\section{DATA AVAILABILITY}

Data presented in this work is available upon reasonable request from Takuo Tanaka.

Received: 24 February 2019; Accepted: 12 September 2019; Published online: 14 October 2019

\section{REFERENCES}

1. Stöckle, R. M., Suh, Y. D., Deckert, V. \& Zenobi, R. Nanoscale chemical analysis by tip-enhanced raman spectroscopy. Chem. Phys. Lett. 318, 131-136 (2000).

2. Anderson, M. S. Locally enhanced Raman spectroscopy with an atomic force microscope. Appl. Phys. Lett. 76, 3130-3132 (2000).

3. Hayazawa, N., Inouye, Y., Sekkat, Z. \& Kawata, S. Metallized tip amplification of near-field Raman scattering. Opt. Commun. 183, 333-336 (2000)

4. Beams, R., Cançado, L. G., Jorio, A., Vamivakas, A. N. \& Novotny, L. Tip-enhanced Raman mapping of local strain in graphene. Nanotechnology 26, 175702 (2015). 
5. Stadler, J., Schmid, T. \& Zenobi, R. Nanoscale chemical imaging of single-layer graphene. ACS Nano 5, 8442-8448 (2011).

6. Park, K. D., Raschke, M. B., Atkin, J. M., Lee, Y. H. \& Jeong, M. S. Probing bilayer grain boundaries in large-area graphene with tip-enhanced raman spectroscopy. Adv. Mater. 29, 1603601 (2017).

7. Lee, C. et al. Unveiling defect-related Raman mode of monolayer $\mathrm{WS}_{2}$ via Tipenhanced resonance raman scattering. ACS Nano 12, 9982-9990 (2018).

8. Iwasaki, T. et al. Local hole doping concentration modulation on graphene probed by tip-enhanced Raman spectroscopy. Carbon 111, 67-73 (2017).

9. Rogalski, J. et al. STM tip-enhanced Raman Spectroscopy and the investigation of doped. Graphene. Vibrational Spectrosc. 91, 128 (2017).

10. Zhang, R. et al. Chemical mapping of a single molecule by plasmon-enhanced Raman scattering. Nature 498, 82-86 (2013).

11. Chiang, N. et al. Confrmational contrast of surface-mediated molecular switches yields ångstrom-scale spatial resolution in ultrahigh vacuum tip-enhanced Raman spectroscopy. Nano Lett. 16, 7774-7778 (2016).

12. Sheng, S. et al. Vibrational properties of a monolayer silicene sheet studied by tipenhanced raman spectroscopy. Phys. Rev. Lett. 119, 196803 (2017).

13. Chen, C., Hayazawa, N. \& Kawata, S. A $1.7 \mathrm{~nm}$ resolution chemical analysis of carbon nanotubes by tip-enhanced Raman imaging in the ambient. Nat. Commun. 5, 3312 (2014).

14. Inouye, Y., Verma, P., Ichimura, T. \& Kawata, S. Near-Field Effects in Tip-enhanced Raman Scattering. In Tip Enhancement 1st edn. (eds Kawata, S., Shalaev, V.) (Elsevier, Amsterdam, 2007).

15. Amorim, B. et al. Novel effects of strains in graphene and other two dimensional. Mater. Phys. Rep. 617, 1-54 (2016).

16. Ikeda, K. et al. Plasmonically nanoconfined light probing invisible phonon modes in defect-free graphene. J. Am. Chem. Soc. 135, 11489-11492 (2013).

17. Ayars, E. J., Hallen, H. D. \& Jahncke, C. L. Electric field gradient effects in Raman spectroscopy. Phys. Rev. Lett. 85, 4180-4183 (2000).

18. Takase, M. et al. Selection-rule breakdown in plasmon-induced electronic excitation of an isolated single-walled carbon nanotube. Nat. Photon. 7, 550-554 (2013).

19. Couto, N. J. G. et al. Random strain fluctuations as dominant disorder source for high-quality on-substrate graphene devices. Phys. Rev. X 4, 041019 (2014).

20. Neumann, C. et al. Raman spectroscopy as probe for nanometer-scale strain variations in graphene. Nat. Commun. 6, 8429 (2015)

21. Mohiuddin, T. M. G. et al. Uniaxial strain in graphene by raman spectroscopy: $G$ peak splitting, grüneisen parameters, and sample orientation. Phys. Rev. B. 79, 205433 (2009)

22. Yasuda, S., Kumagai, R., Nakashima, K. \& Murakoshi, K. Electrochemical potential stabilization of reconstructed $\mathrm{Au}(111)$ structure by monolayer coverage with graphene. J. Phys. Chem. Lett. 6, 3403-3409 (2015).

23. Ferrari, A. C. \& Basko, D. M. Raman spectroscopy as a versatile tool for studying the properties of graphene. Nat. Nanotechnol. 8, 235-246 (2013).

24. Posonov, Y. S., Ushakov, A. V. \& Streltsov, S. V. Electronic Raman scattering in graphite and single-layer and few layer graphene. Phys. Rev. B 91, 195435 (2015).

25. Wang., Y. Y. et al. Raman studies of monolayer graphene: the substrate effect. J. Phys. Chem. C. 112, 10637-10640 (2008).

26. Wang, W. X. et al. The study of interaction between graphene and metals by Raman spectroscopy. J. Appl. Phys. 109, 07C501 (2011).

27. Oznuluer, T. et al. Synthesis of graphene on gold. Appl. Phys. Lett. 98, 183101 (2011).

28. Lee, J. E., Ahn, G., Shim, J., Lee, Y. S. \& Ryu, S. Optical separation of mechanical strain from charge doping in graphene. Nat. Commun. 3, 1024 (2012).

29. Nie, S. et al. Scanning tunneling microscopy study of graphene on Au(111): growth mechanisms and substrate interactions. Phys. Rev. B 85, 205406 (2012).

30. Itoh, T., Yamamoto, Y. K. \& Ozaki, Y. Plasmon-enhanced spectroscopy of absorption and spontaneous emissions explained using cavity quantum optics. Chem. Soc. Rev. 46, 3904 (2017).

31. Frank, O. et al. Compression behavior of single-layer graphenes. ACS Nano 4, 3131-3138 (2010).

32. Frank., O. et al. Raman 2D-band splitting in graphene: theory and experiment. ACS Nano. 5, 2231-2239 (2011).

33. Das, A. et al. Monitoring dopants by Raman scattering in an electrochemically top-gated graphene transistor. Nat. Nanotechnol. 3, 210-215 (2008).

34. Froehlicher, G. \& Berciaud, S. Raman spectroscopy of electrochemically gated graphene transistors: geometrical capacitance, electron-phonon, electron-electron, and electron-defect scattering. Phys. Rev. B 91, 205413 (2015).

35. Bruna, M. et al. Doping dependence of the raman spectrum of defected graphene. ACS Nano 8, 7432-7441 (2014).

36. Pisana, S. et al. Breakdown of the adiabatic born-oppenheimer approximation in graphene. Nat. Mater. 6, 198-201 (2007)

37. Mueller, N. S. et al. Evaluating arbitrary strain configurations and doping in graphene with Raman spectroscopy. 2D Mater. 5, 015016 (2018).
38. Metten, D., Federspiel, F., Romeo, M. \& Berciaud, S. All-Optical blister test of suspended graphene using micro-Raman spectroscopy. Phys. Rev. Appl. 2, 054008 (2014).

39. Sławińska, J., Dabrowski, P. \& Zasada, I. Doping of graphene by a Au(111) substrate: calculation strategy within the local density approximation and a semiempirical van der waals approach. Phys. Rev. B 83, 245429 (2011).

40. Klusek, Z. et al. Graphene on gold: electron density of states studies by scanning tunneling spectroscopy. Appl. Phys. Lett. 95, 113114 (2009).

41. $\mathrm{Wu}, \mathrm{Y}$. et al. Tuning the doping type and level of graphene with different gold configurations. Small 8, 3129-3136 (2012).

42. Marchenko, D. et al. Giant rashba splitting in graphene due to hybridization with gold. Nat. Commun. 3, 1232 (2012).

43. Arora, A. K., Rajalakshmi, M., Ravindran, T. R. \& Sivasubramanian, V. Raman spectroscopy of optical phonon confinement in nanostructured materials. J. Raman Spectrosc. 38, 604-617 (2007).

44. Banszerus, L. et al. Ultrahigh-mobility graphene devices from chemical vapor deposition on reusable copper. Sci. Adv. 1, e1500222 (2015).

45. Ding, F. et al. Stretchable graphene: a close look at fundamental parameters through biaxial straining. Nano Lett. 10, 3453-3458 (2010).

46. Malard, L. M., Pimenta, M. A., Dresselhaus, G. \& Dresselhaus, M. S. Raman spectroscopy in graphene. Phys. Rep. 473, 51-87 (2009).

47. Ferralis, N. Probing mechanical properties of graphene with Raman spectroscopy. J. Mater. Sci. 45, 5135-5149 (2010).

48. Mohr, M., Mautlzsch, J. \& Thomsen, C. Splitting of the Raman 2D band of graphene subjected to strain. Phys. Rev. B. 82, 201409 (2010).

49. Samsonov, G. Handbook of Physicochemical Properties of the Elements. (IFI Plenum, New York, 1968).

50. Beversluis, M. R., Bouhelier, A. \& Novotny, L. Continuum generation from single gold nanostructures through near-field mediated intraband transitions. Phys. Rev. B 68, 115433 (2003).

51. Pratama, F. R., Ukhtary, M. S. \& Saito, R. Non-vertical optical transition in near-field enhanced spectroscopy of graphene. J. Phys.: Condens. Matter 31, 265701 (2019).

52. Basko, D. M. Boundary problems for dirac electrons and edge-assisted Raman scattering in graphene. Phys. Rev. B 79, 205428 (2009).

53. Yang, B., Kazuma, E., Yokota, Y. \& Kim, Y. Fabrication of sharp gold tips by threeelectrode electrochemical etching with high controllability and reproducibility. $J$. Phys. Chem. C. 122, 16950-16955 (2018).

54. Yan, J. A., Ruan, W. Y. \& Chou, M. Y. Phonon dispersion and vibrational properties of monolayer, bilayer, and trilayer graphene: density-functional perturbation theory. Phys. Rev. B 77, 125401 (2008).

\section{ACKNOWLEDGEMENTS}

We are grateful to Professor Riichiro Saito for the helpful discussion on the activation mechanism of the forbidden modes. The authors would also like to thank the Synthetic Organic Chemistry Laboratory in RIKEN for the usage of the RAMAN-11 facility with the help of Dr. Francesca Celine Catalan from the Surface and Interface Science Laboratory also in RIKEN. This work was financially supported in part by the Grant-in-aid for scientific research (KAKENHI) no. $15 \mathrm{H} 03569$ (N.H.) and RIKEN Center for Advanced Photonics.

\section{AUTHOR CONTRIBUTIONS}

M.V.B., K.I. and N.H. designed the STM-TERS experiments. M.V.B. conducted the experiments and analysed the data. M.V.B. and Y.Y. prepared the Au(111) substrates. S.Y. made the $\mathrm{Gr} / \mathrm{Au}(111)$ samples. B.Y, E.K. and Y.K. designed the tip fabrication experiments. T.T. supervised the work. The paper was written through contributions of all authors. All authors have given approval to the final version of the paper.

\section{COMPETING INTERESTS}

The authors declare no competing interests.

\section{ADDITIONAL INFORMATION}

Supplementary information is available for this paper at https://doi.org/10.1038/ s41699-019-0121-7.

Correspondence and requests for materials should be addressed to M.V.B., N.H. or T.T.

Reprints and permission information is available at http://www.nature.com/ reprints 
Publisher's note Springer Nature remains neutral with regard to jurisdictional claims in published maps and institutional affiliations.

cc) (i) Open Access This article is licensed under a Creative Commons Ar Attribution 4.0 International License, which permits use, sharing, adaptation, distribution and reproduction in any medium or format, as long as you give appropriate credit to the original author(s) and the source, provide a link to the Creative
Commons license, and indicate if changes were made. The images or other third party material in this article are included in the article's Creative Commons license, unless indicated otherwise in a credit line to the material. If material is not included in the article's Creative Commons license and your intended use is not permitted by statutory regulation or exceeds the permitted use, you will need to obtain permission directly from the copyright holder. To view a copy of this license, visit http://creativecommons. org/licenses/by/4.0/.

(c) The Author(s) 2019 